\title{
Clinical Impacts of Oral Gastrographin in Adhesive Intestinal Obstruction
}

\author{
Khaled Mohamed Sharaf, MD; ${ }^{1}$ Hisham Omran, MD. ${ }^{2}$
}

\author{
1) Department of General Surgery, Zagazig University, Egypt. \\ 2) Department of General Surgery, Ain Shams University, Egypt.
}

Background: Orally administered gastrographin has been used for early resolution of postoperative small bowel obstruction (POSBO) and to reduce the need for surgery in various studies.

Aim of the work: We carried out the study to evaluate the efficacy of gastrographin in patients with partial small bowel obstruction.

Patients and methods: A prospective study was done. Patients with suspected strangulations, complete obstruction, obstructed hernia, bowel malignancy and radiation enteritis were excluded. One hundred (100) patients with partial adhesive small bowel obstruction were given an initial trial of conservative management for 48 hours. Sixty patients improved within 48 hours and the other forty patients were given $100 \mathrm{ml}$ of undiluted gastrographin through the nasogastric tube. In 38 patients the contrast reached the colon within $24 \mathrm{hs}$. In the remaining two patients the contrast failed to reach the colon and these underwent surgery.

Results: The use of gastrographin avoided surgical intervention in 95\% (38 of 40) of patients who failed conservative management of POSBO. Gastrographin also decreased the overall requirement for surgical management of POSBO to $5 \%$ (2 of 100).

Conclusion: The use of gastrographin in patients with partial POSBO helps in resolution of symptoms and avoid the need for surgical management in the majority of patients.

Key words: $P O S B O$, adhesive, small bowel, gastrographin, intestinal obstruction.

\section{Introduction:}

Intestinal obstruction is a partial or complete blockage to the passage of intestinal contents through the gastrointestinal tract and is a major indication for urgent abdominal surgery. Unless treated properly, intestinal obstruction can be associated with high morbidity and mortality rates. Postoperative abdominal adhesions represent the main etiological factor for intestinal obstruction, followed by neoplasms and hernias. Although the mortality rate from intestinal obstruction has previously been quoted at $30-50 \%$, better diagnostic and treatment methods could potentially reduce this to $5-10 \%$.

Small bowel obstruction can be classified according to completeness: Partial vs. Complete (or high grade vs. low grade), according to etiology: Adhesive vs.
Non-adhesive, according to timing: Early vs. Late ( $>30$ days after surgery). The most important risk factor for adhesive SBO is the type of surgery and extent of peritoneal damage. Surgeries of the colon and rectum are associated with a higher risk of adhesionrelated problems. ${ }^{1}$

Adhesive obstructions that require surgical intervention can be associated with serious complications when surgery for the obstruction is delayed $>48 \mathrm{hs}$. The mortality rate, which is only around $5 \%$ in uncomplicated obstruction, rises to $30 \%$ in patients with strangulation of the small intestine. The most effective way to reduce the mortality and morbidity caused by postoperative adhesions, therefore, through early diagnosis and appropriate treatment.

Adhesive small bowel obstruction 
(POSBO) occur in $6 \%$ to $30 \%$ of patients who have undergone a previous laparotomy and is responsible for a large number of emergency surgical admissions. Surgical intervention is required in $20 \%$ to $30 \%$ of cases, whereas conservative management is sufficient in majority of case. ${ }^{2}$

Some surgeons suggest conservative management for up to 5 days provided that no obvious signs of intestinal strangulation are present. On the other hand, it has been suggested that a delay in surgical intervention of more than 24 hours increases complication rates and prolongs postoperative hospital stay. Neither complete nor incomplete small bowel obstruction can be reliably identified clinically or with plain radiological studies. ${ }^{3}$

The initial management of patients with complete SBO remains controversial. Although complete SBO is associated with a higher requirement for small-bowel resection $(31 \%)$ in some series, others have demonstrated that non-operative management is still successful in $41 \%$ to $73 \%$ of patients with complete obstruction. ${ }^{4}$

In the absence of any evidence of gangrene or bowel strangulation, trial of conservative management results in the resolution of obstruction in $70-80 \%$ of cases as a significant number of these episodes are of low-grade partial obstruction. Most patients who ultimately require surgery have high-grade partial obstruction or complete obstruction. ${ }^{5}$

Gastrographin is a water-soluble contrast medium of high osmolarity composed of sodium diatrizoate, meglumine amidotrizoate and wetting agent (polysorbate 80 ).

The value of gastrographin was early detection of patients who could be managed non-operatively. Gastrographin may also have therapeutic effect in adhesive small bowel obstruction because of its hyperosmolarity. Gastrographin promotes shifting of fluid into the bowel lumen and increases the pressure gradient across the obstructing site. The bowel contents are diluted and in the presence of wetting agent, easier passage of the bowel contents through the narrowed lumen is allowed, gastrographin decreases bowel wall oedema and enhances bowel motility.
Furthermore, gastrographin is water soluble and relatively safe even if the obstruction is complicated by perforation. Gastrographin may also shorten postoperative ileus and relieve intestinal obstruction caused by impacted ascaris lumbricoids and bezoar. ${ }^{6}$

\section{Patients and methods:}

One hundred patients were included in this study at the Department of General Surgery, Alazhar hospital in Riyadh, Saudi Arabia, and Ain Shams university hospitals in Cairo, Egypt from April 2012 to January 2014. Patients with postoperative small bowel obstruction who were admitted to our department were considered for inclusion in this study.

The diagnosis of small bowel obstruction was based on the history of colicky abdominal pain associated with abdominal distension, nausea, vomiting and obstipation, history of prior laparotomy and characteristic features of small bowel obstruction on plain abdominal radiograms.

Exclusion criteria included patients whose X-rays showed classical features of obstruction in the absence of any colonic gas were considered to have complete obstruction, patients with clinical diagnosis indicating strangulation or gangrene of the gut and patients with obstructed abdominal wall hernia, known inflammatory bowel disease, radiation enteritis and intra-abdominal malignancy were excluded.

The patients were managed conservatively with nasogastric tube decompression, hydration and correction of any electrolyte imbalance for 48 hours.

Abdominal radiograph at admission was evaluated for the number of air fluid levels and the diameter of dilated loops were recorded. Serial follow-up X-rays were taken at 4, 8, 16, 24, 36 and $48 \mathrm{hrs}$.

Clinical improvement was defined as a decrease in abdominal distension and pain and nasogastric tube output less than $250 \mathrm{ml} / 24 \mathrm{~h}$ associated with passage of flatus or stool. Radiological improvement was considered when the number of dilated loops and air fluid levels decreased to less than 3 
and the diameter of bowel loops decreased. The conservative management was continued beyond $48 \mathrm{hs}$ in patients who showed clinical or radiological improvement or both until complete resolution of symptoms and resumption of oral diet.

Patients who failed to improve despite of $48 \mathrm{hs}$ of conservative treatment were given $100 \mathrm{ml}$ gastrographin through the nasogastric tube and were followed up clinically and with serial radiographs at 4, 8, 16 and 24 hrs. Patients in whom contrast failed to reach the colon in 24 hs were considered to have complete obstruction and were planned for surgery. While patients in whom contrast has reached the colon within 24 hours were followed until the resolution of symptoms.

Statistical analysis:

Data were collected, coded, revised and entered to the Statistical Package for Social Science (IBM SPSS) version 21. The qualitative data were presented as frequencies and percentages.

\section{Results:}

Between April 2012 to January 2014, 100 patients presented with mechanical intestinal obstruction at Alazhar hospital in Riyadh, Saudi Arabia and Ain Shams University Hospital Cairo, Egypt. These consisted of 60 men and 40 women with age range of 20-60 years.

The duration of symptoms ranged between 2-5 days. The commonest surgical procedures involved small and large bowel and gynecological operations Table (1).

The 24 hours output of the nasogastric tube was $600-1200 \mathrm{ml}$. Plain abdominal radiogram showed four to eight air fluid levels.

All 100 patients were managed conservatively with intravenous fluids, nasogastric decompression and correction of electrolytes and acid base balance.

60 patients improved on conservative management within 48 hs. None of these patients who showed improvement on conservative treatment after 48 hours relapsed. All patients were able to tolerate soft diet by 4 days.
40 patients failed to improve clinically or radiologically after 48 hours of conservative management. These patients were given 100 $\mathrm{ml}$ of gastrographin via the nasogastric tube after complete suction of the gastric fluid, the tube was then clamped for 2 hours and serial X-rays were taken. In 38 patients (95\%) the contrast reached the colon within 24 hours and they were managed conservatively.

In 2 patients $(5 \%)$ no contrast was seen in the colon after 24 hours and these patients underwent surgery. Both had adhesions between the loops of the intestine which were lysed. They have smooth postoperative course and they were discharged on the $5^{\text {th }}$ and $6^{\text {th }}$ postoperative days.

The use of gastrographin thus avoided the need for surgery in $95 \%$ of patients who failed conservative treatment of adhesive small bowel obstruction.

Gastrographin shortened the duration of obstruction and hospital stay. The time from the hospital admission for obstruction to resolution of symptoms was significantly lower with the use of gastrographin.

\section{Discussion:}

Postoperative small bowel obstruction is a common cause of surgical admission. In the absence of bowel strangulation initial trial of conservative is given to most patients, especially those suspected to partial small bowel obstruction based on established clinical and radiological criteria. In various published reports the rate of operative management of adhesive obstruction has been variable (27$42 \%$ ) as both partial and complete obstruction were included. ${ }^{7}$ Successful response to nonoperative treatment is replaced to be $73 \%$ to $90 \% .8$

Controversy still exists regarding the reasonable period of time for conservative treatment before resorting to surgical intervention in patients with ASBO. It has been reported that non-operative management up to 5 days duration can be used safely for most patients, although some reports suggest a shorter period of 24 or 48 to 72 hours. ${ }^{9}$

Gastrographin is a water-soluble gastrointestinal radiologic contrast medium 
that has a very high osmolarity. The osmolarity of this undiluted contrast material is $1900 \mathrm{~m} \mathrm{Osm} / \mathrm{L}$, approximately six times that of extracellular fluid, ${ }^{10}$ its hyperosmolarity draws fluid from intravascular and extracellular spaces into the lumen, promoting proximal bowel distension and this increases the gradient pressure across the obstructing segment. Moreover, dilution of the bowel contents, combined with the effect of the wetting agent, may allow easier slipper passage through narrowed area, decreased localized bowel wall oedema and enhanced peristalsis are additional possible contributing factors. ${ }^{11}$ Gastrographin normally reaches cecum in healthy subjects in $45 \mathrm{~min}$ (30$90 \mathrm{~min}$ ). Thus it has a predictive role in management of small bowel obstruction. ${ }^{12}$

Chen et al. found that contrast in the colon within 24 hours obviated the need for surgery in patients with adhesive obstruction. Absence of contrast in the colon after 24 hours may thus be a better way of differentiating complete from partial episodes of POSBO. ${ }^{13}$

Assalia et al. ${ }^{8}$ and Biondo et al. ${ }^{14}$ reported that the use of gastrographin resulted in quicker resolution of obstructive episode and decreased the duration of hospitalization. 8,14

Feigin et al. found no therapeutic benefit of gastrographin in POSBO. However in this study no distinction was made between partial or complete obstruction which might have led to poor results. ${ }^{15}$

Choi et al. ${ }^{16}$ found that gastrographin reduced the need for surgery by $74 \%$ when used in patients with partial obstruction. ${ }^{16}$

In the meta-analysis conducted by Abbas et al., 17 it was reported that the passage of gastrographin in the colon within $24 \mathrm{hrs}$ predict the resolution with specificity of $96 \%$ and sensitivity of $97 \%$. In addition, found that gastrographin did not reduce the need of surgical intervention but reduced hospital stay for patients did not require surgery. ${ }^{17}$

In addition al patients included in our study received an initial conservative trial of $48 \mathrm{~h}$, a significant number of partial obstruction resolve spontaneously with bowel decompression, thus avoiding unnecessary use of gastrographin.
In our study, only 40 patients of 100 patients required the use of gastrographin. Further, by strict inclusion of patients with partial obstruction, based on radiological criteria, the chances of delay of surgical treatment in the case of strangulation or complete obstruction is reduced to minimum. Only two patients in our study were thought to have partial obstruction on plain abdominal X-rays turned out to have complete obstruction that did not respond to gastrographin. In both these patients there was no evidence of strangulation or gangrene at surgery.

In our study, the use of gastrographin avoided the need for surgery in $95 \%$ of patients who failed conservative management of PoSBO. Gastrographin also decreased the need for surgical management of PoSBO to $5 \%$. Since we only included patients with partial obstruction, the actual need for surgical intervention after failure of conservative management might have been lower in our patients as compared to the published reports.

\section{Conclusion:}

One can conclude that orally administered gastrographin is safe and may have a therapeutic role in POSBO as it helps in its resolution and shortens the hospital stay. Gastrographin also provides clues for early diagnosis of patients who require surgery and perhaps decreases the need for such an operation.

\section{Reference:}

1- Parker MC, Ellis H, Moran BJ: Postoperative adhesions: ten-year follow-up of 12,584 patients undergoing lower abdominal surgery. Dis Colon Rectum 2001; 44(6): 822-829.

2- Seror D, Feigen E, Szold A: How conservatively can postoperative small bowel obstruction be treated? Am J Surg 1993; 165: 121-125.

3- Ha HK, Kim JS, Lee HJ: Differentiation of simple and strangulated small bowel obstruction: Usefulness of known CT criteria. Radiology 1997; 204: 507-512.

4- Tanaka S, Yamamoto T, Kubota D: Predictive factors for surgical indication in adhesive small bowel obstruction. Am J Surg 2008; 196: 23-27.

5- Brolin RE: Partial small bowel obstruction. 

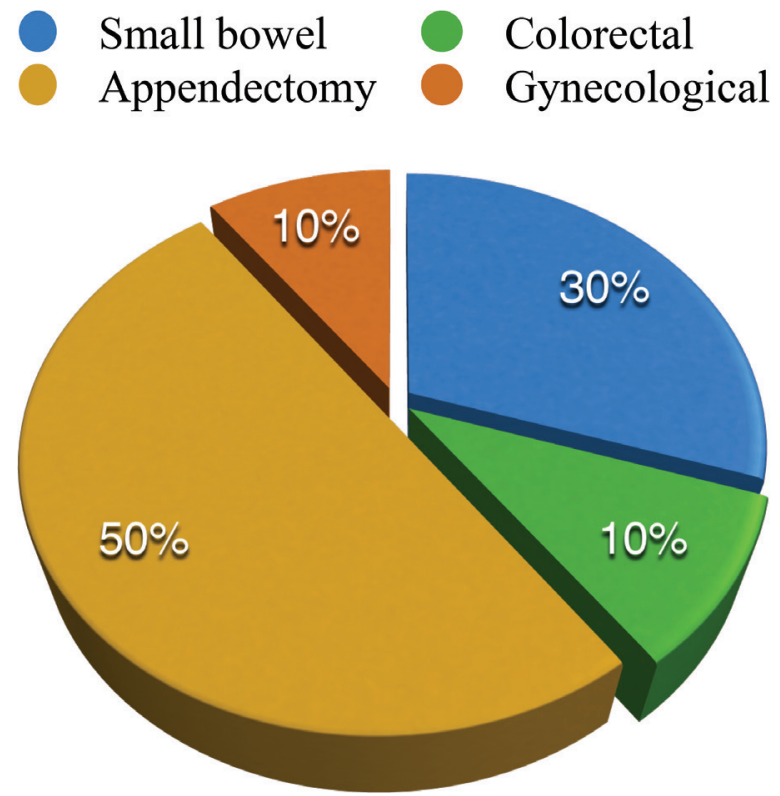

Figure (1): Nature of previous surgical procedures.
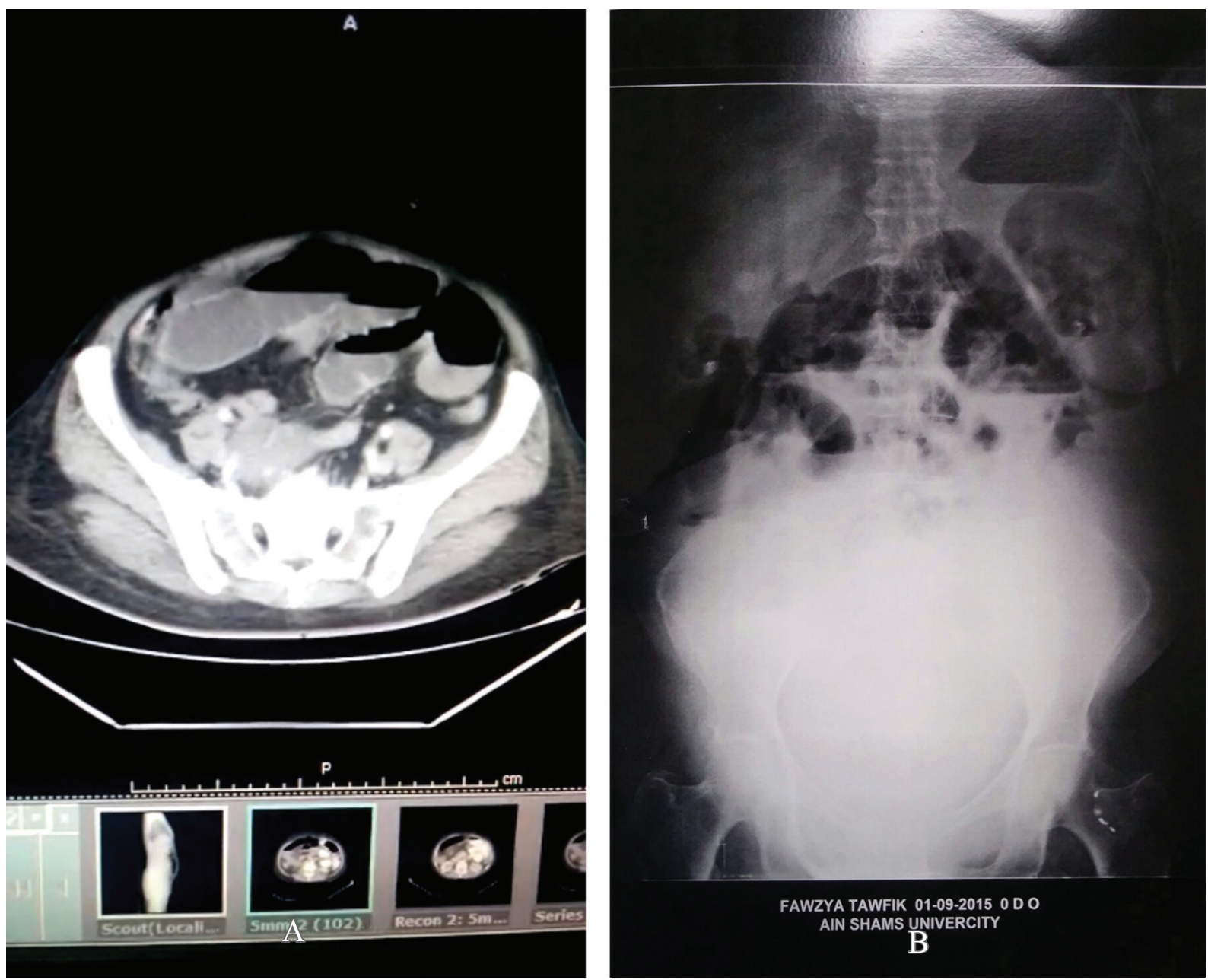

Figure (2): A) CT scan showing dilated bowel loops (IO) and B) AXR Abdomen showing multiple fluid levels (IO).

Surgery 1984; 95: 145-149.

6- Hamid A, Naif IA, Khairi AH, Hazem MZ, Abdulmohsen AA, Faten OA: Role of water soluble contrast agent in assigning patients to a non-operative course in adhesive small bowel obstruction. Oman Med J 2011; 26(6): 


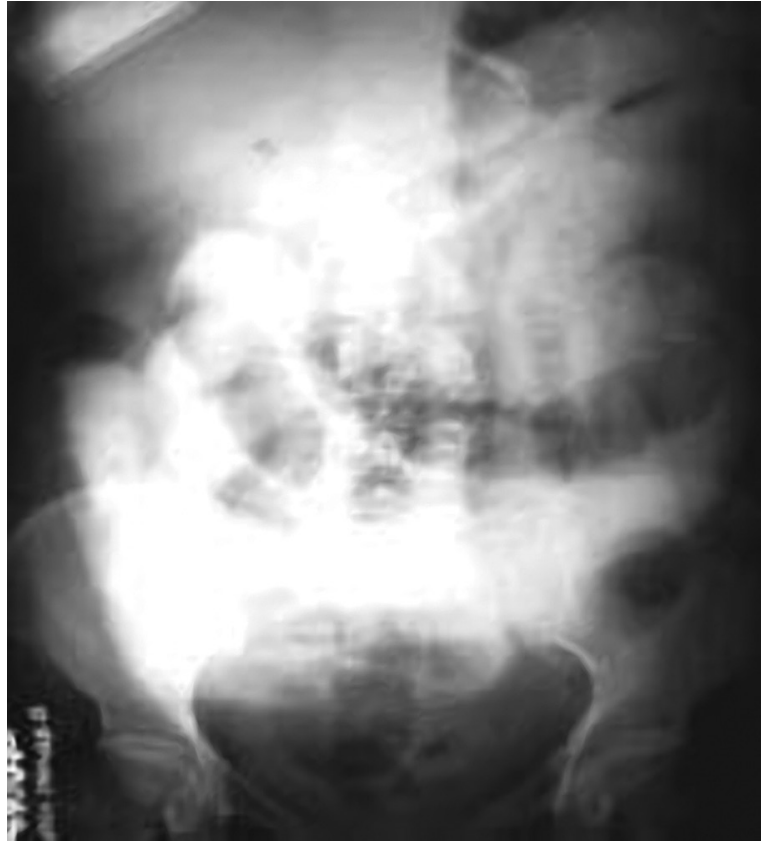

Figure (3): AXR at 20 hrs dye not reaching the caecum.

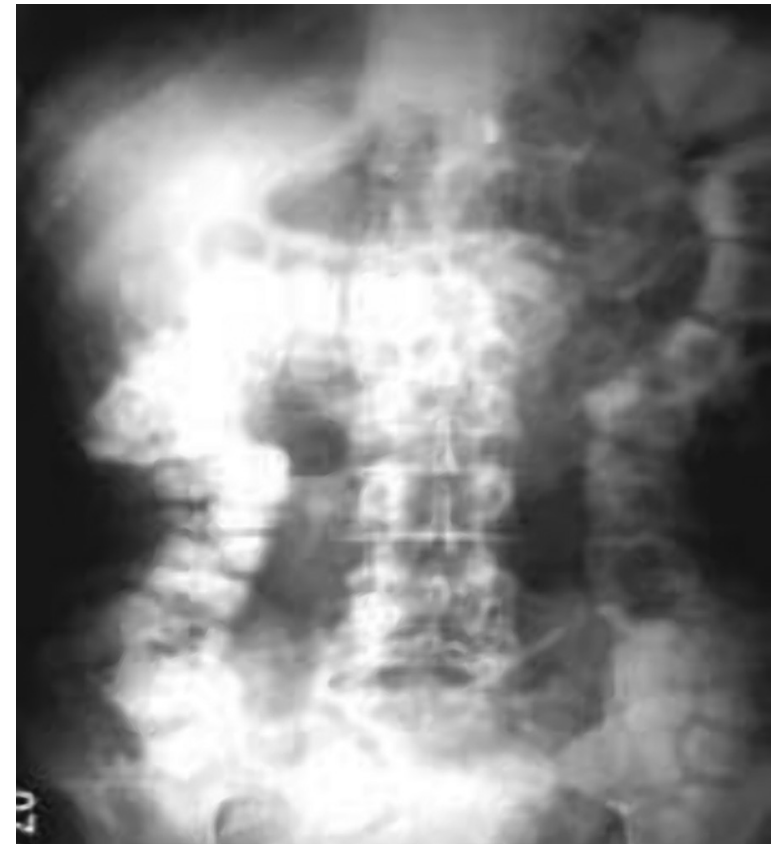

Figure (4): AXR at 20 hrs showing dye within the colon.

\section{Table (1): Nature of previous surgical procedures}

\begin{tabular}{|l|l|l|}
\hline Surgical procedures & No. of patients & $\%$ \\
\hline Small bowel & 30 & 30 \\
\hline Colorectal & 10 & 10 \\
\hline Appendectomy & 50 & 50 \\
\hline Gynecological & 10 & 10 \\
\hline
\end{tabular}

454-456.

7- Chen SC, Chang KJ, Lee PH: Oral urografin in postoperative small bowel obstruction. World J Surg 1999; 23(10): 1051-1054.

8- Assalia A, Schein, Lopelman D: Therapeutic effect of oral Gastrographin in adhesive partial small bowel obstruction: A prospective randomized trial. Surgery 1994; 115: 433-437.

9- Feigen E, Seror D, Szold A: Water-soluble contrast medium has no therapeutic effect on postoperative small bowel obstruction: Results of a prospective randomized clinical trial. Am J Sug 1996; 171-227.

10- Mohamed F, Amir F, Ayman E, Elyamani F, Tito E, Mohamed Y, Waleed O: Clinical impacts of oral Gastrographin follow through an adhesive small bowel obstruction (SBO). Journal of Surgical Research 2010; 162: 170-176.

11- Strodahl A, Laerum F, Gjolberrg T: Watersoluble contrast media in radiology of small bowel obstruction. Acta Radiol 1988; 29: 53-56.

12- Sorabh K, Gaurav J, Ajit S, Sandesh S, Kailash P, Subodh V: Prospective evaluation of oral Gastrographin in postoperative small bowel obstruction. Journal of Surgical Research 2006; 131: 256-260.

13- Chen SC, Lin FY, Lee PH: Water-soluble contrast study predicts the need for early surgery in adhesive small bowel obstruction. B J Surg 1998; 85: 1692-1694.

14- Biondo S, Pares D, Mora L: clinical study of Gastrographin administration in patients with adhesive small bowel obstruction. $B J$ Surg 2003; 90: 542-546.

15- Bar-Maor JA, de Carvalho JL, Chappell $\mathrm{J}$ : Gastrographin treatment of intestinal obstruction due to Ascaris Lumbricoides. $J$ Pediatr Surg 1984; 19: 174-176.

16- Choi HK, Chu KW, Law WL: Therapeutic value of Gastrographin in adhesive small bowel obstruction after unsuccessful 
conservative treatment: A prospective randomized trial. Ann Surg 2002; 236-241.

17- Abbas S, Bisset IP, Parry BR: Analysis of oral water soluble contrast agent in the management of adhesive small bowel obstruction. Br J Surg 2007; 94: 404-411. 


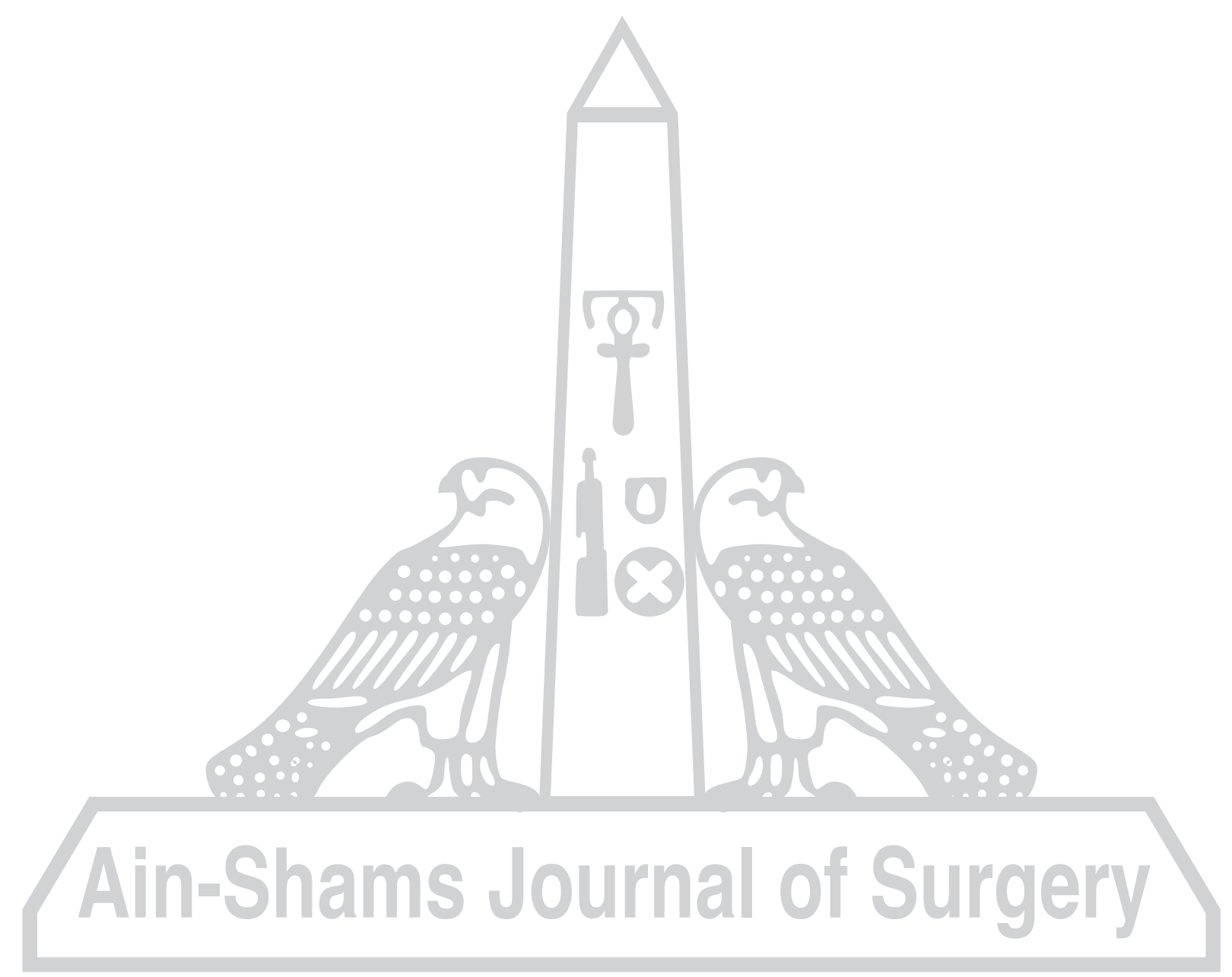

